\title{
The LHCb muon detector commissioning and first running scenarios
}

\author{
S. Furcas *, A. Sarti \\ Laboratori Nazionali di Frascati, Frascati, Italy
}

\section{A R T I C L E I N F O}

Keywords:

Tracking detectors

GEM

Micro-pattern gas detectors

\begin{abstract}
A B S T R A C T
The LHCb muon detector, part of the first LHCb trigger level (LO), has been built to provide a fast and efficient identification of the muons produced in pp collisions at the LHC. The expected performances are: 95\% L0 trigger efficiency within a $25 \mathrm{~ns}$ time window and muon identification in L0 with a $p_{T}$ resolution of $20 \%$. The detector has been built using Multi-Wire Proportional Chambers and Gas Electron Multiplier technology. The chambers are arranged in five stations, interspersed with iron filters placed along the beam pipe. The results obtained in the commissioning of all the installed chambers and the measured performances are presented. The strategies foreseen for the detector calibration, the results of the space and time alignment efforts and few first running scenarios are discussed.
\end{abstract}

(c) 2009 Elsevier B.V. All rights reserved.

\section{Introduction}

The LHCb muon system [1] is composed of five muon stations (M1-M5), equipped with 1368 Multi-Wire Proportional Chambers (MWPC) and 24 Gas Electron Multipliers (GEM) chambers (used in the inner region of $\mathrm{M} 1$ ), interspersed with iron filters. Each station is subdivided into four regions (R1-R4) with readout granularity decreasing according to the distance from the proton interaction point (IP). The readout geometry has been optimized in order to maximize the muon system main task performances: level zero (L0) muon trigger efficiency, resolution of online measurement of the muon transverse momentum $\left(p_{T}\right)$ and $\mu$ offline identification efficiency.

The detector requirements are: a high ( $99 \%)$ track hit detection efficiency into the bunch crossing time window ( $25 \mathrm{~ns}$ ) and time resolution better than $4 \mathrm{~ns}$. A good space resolution is also needed to provide a $p_{T}$ resolution less than $20 \%$. In this paper we report the results obtained so far for the installed stations using data from cosmic rays and beam events comparing them to the test bench and test beam data (see Section 1). Strategies for the calibrations and detector monitoring in the first experiment running are also discussed (see Section 2).

\section{Commissioning of the muon system}

Since spring 2008, the M2-M5 stations are fully installed and under test at the reduced voltage of $2.5 \mathrm{kV}$ (the chambers working point is $2.5-2.65 \mathrm{kV}$, depending on the chamber type) with an

\footnotetext{
* Corresponding author.

E-mail address: sara.furcas@lnf.infn.it (S. Furcas).
}

$\mathrm{Ar} / \mathrm{CO}_{2} 40 / 60$ gas mixture (the final mixture will be $\left.\mathrm{Ar} / \mathrm{CO}_{2} / \mathrm{CF}_{4} 40 / 55 / 5\right)$. The $99.7 \%$ of the $4416 \mathrm{HV}$ gas gaps are working with a stable dark current below $10 \mathrm{nA}$.

The ongoing commissioning activities are mainly devoted to the debug of problematic (noisy, unresponsive) electronic channels $(<0.1 \%)$, the time alignment of all the readout channels, the measurement of track/hit time resolution and of chamber efficiencies. While the first two tasks can be accomplished by custom software and hardware test system, the evaluation of timing and efficiency performances needs to be performed on data from cosmic runs and from first beam tests.

In order to monitor the status of the large amount of electronics channels $(\sim 120 \mathrm{k})$ a procedure based on the acquisition of noise counts at different threshold is used. Noisy and dead or unresponsive channels can be easily spotted and fixed, while from the acquired noise spectra each channel can be properly characterized and configured [2]. The time alignment of the FEE channels is performed using a dedicated pulsing system controlled by the online experiment control system (ECS) and its quality has been checked using beam dump events. Recorded events have been analyzed in order to check the occupancy plots and spot problematic channels.

The chamber efficiency has been measured using tracks selected requiring three hits in three different muon stations and looking for the fourth. An efficiency of $99 \%$ is measured for chambers of all the different regions when properly taking into account the tracks projectivity (for those not coming from the IP). The chamber alignment is performed by using tracks reconstructed using a Neural Net (NN) reconstruction tool selected by the requirement that they have at least three hits in the muon stations. In order to measure their alignment, two stations were kept fixed, while the other two were left free to move and rotate. By asking that 2 out of the 3(4) hits/tracks were falling in the fixed 


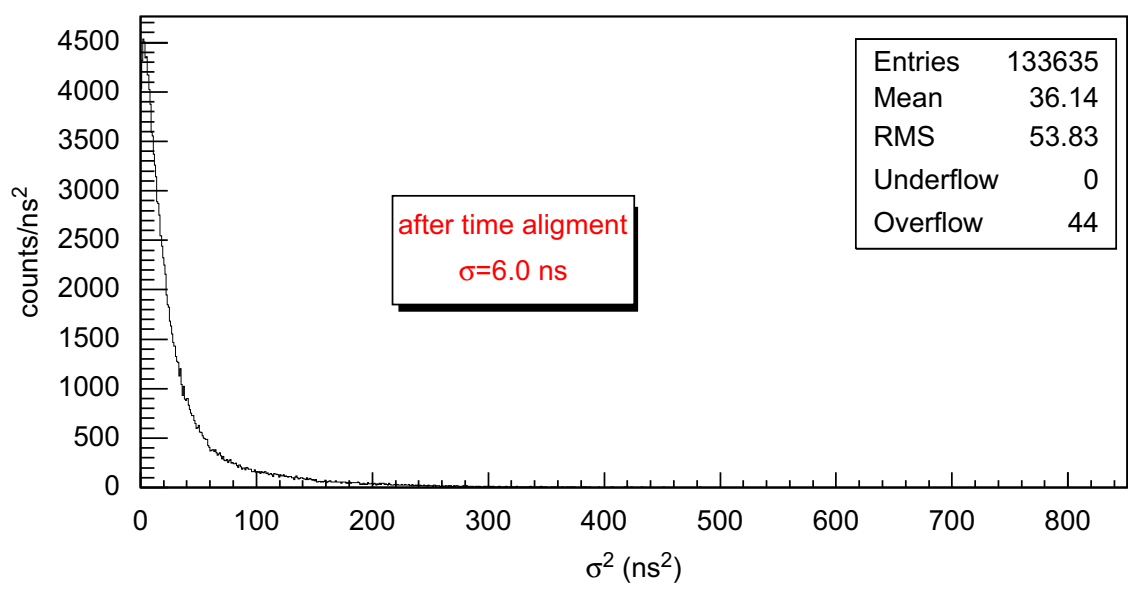

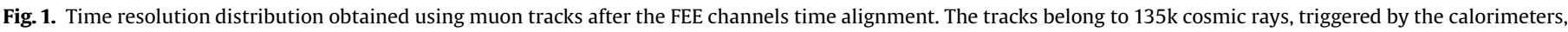
collected in the end of 2008 .

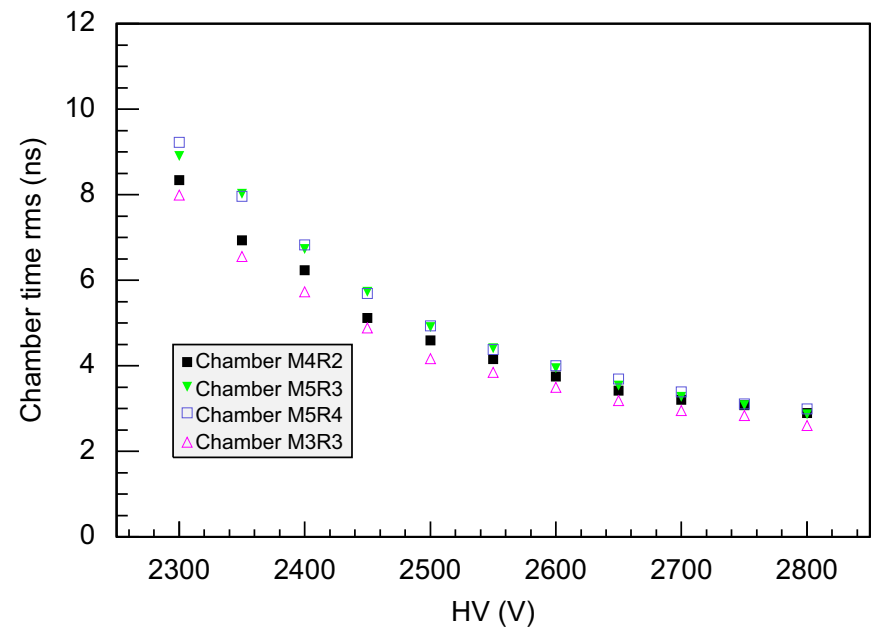

Fig. 2. Time resolution of four different type MWPC chambers as a function of the applied high voltage, obtained from the analysis of data collected on a cosmic ray test bench.

stations, the residuals have been minimized using the remaining hit(s). Results obtained with offline analysis agree with measurements from survey probes in the experiment hall.

The chambers time resolution has been measured using tracks built up using the muon system alone, from a data sample of $135 \mathrm{k}$ cosmic rays triggered by the $\mathrm{LHCb}$ calorimeters, collected in the end of 2008. The tracks were made using a NN algorithm and were required to have at least three hits in three different muon stations. The time residuals were then minimized on the track sample with an iterative process. The obtained results on a sample for muon tracks are shown in Fig. 1. The measured time resolution ( $\sim 6 \mathrm{~ns}$ ) is in good agreement with what expected from cosmic rays analysis in test bench for an operating voltage of $2.5 \mathrm{kV}$ (see Fig. 2).

\section{First running scenarios}

The online monitoring and calibration procedures to be made on first data acquired are of fundamental importance in order to ensure a properly working system and maximize the detector physics performances (trigger rate, quality of selected events).

One of the crucial tasks performed by the muon system where calibration plays a fundamental role is the identification of muon

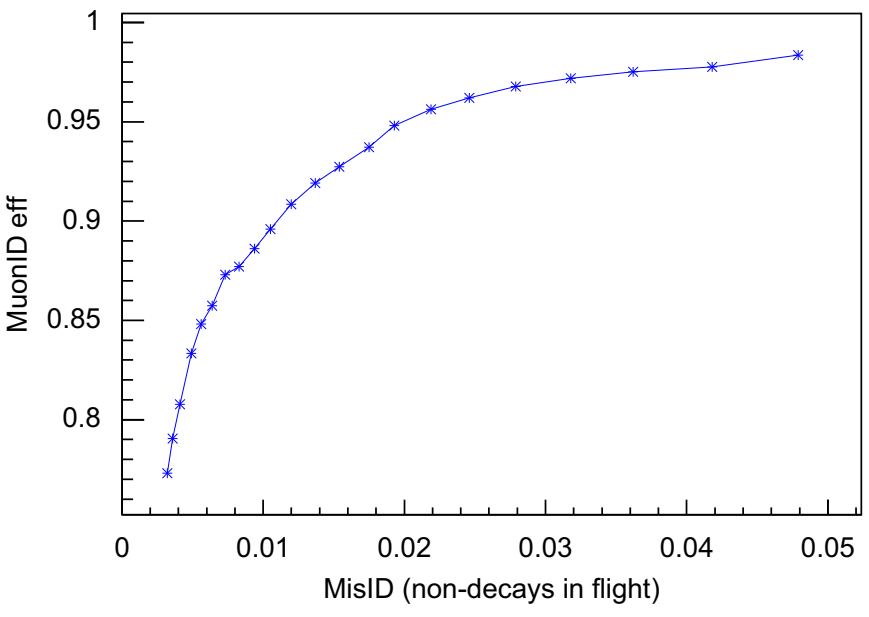

Fig. 3. Muon identification and misID efficiency for different DLL cuts. The misID has been evaluated considering only the component due to hadrons not decaying in flight.

tracks. The muon identification [3] consists of two main steps. Firstly, hits in the muon stations are searched around the track extrapolation in a Field of Interest (FOI) that is function of the tracks momentum. Then, a $\chi^{2}$ like variable (DLL) is built using the hits distance with respect to the track extrapolation.

The calibration, and monitoring, of the algorithm performances will be performed using control data samples selected using only track quality and kinematics/vertex cuts. Two specific decay channels were studied in detail: the $\mathrm{J} / \Psi \rightarrow \mu \mu$ originating from $B$ decays and the $\Lambda \rightarrow p \pi$ decays. They are abundant (can be used in a low luminosity environment), have a clean kinematic signature and are selected with high efficiency by the LHCb trigger.

The $\mathrm{J} / \Psi \rightarrow \mu^{+} \mu^{-}$events used in the analysis are those triggered by a single- $\mu$ line: the muon not used by the trigger can thus be used for the calibration procedure after having applied momentum, transverse momentum and track quality $\left(\chi^{2} / n D o F<3\right)$ cuts. The $\mathrm{J} / \psi$ candidate is also selected by requiring a reconstructed invariant mass in a $100 \mathrm{MeV} / \mathrm{c}^{2}$ window and a good quality vertex $\left(\chi^{2} / n D o F<3\right)$. The $\Lambda \rightarrow \mathrm{p} \pi$ events are selected applying track momentum and transverse momentum cuts. The $\Lambda$ candidate is thus required to have a good vertex quality $\left(\chi^{2} / n D o F<\right) 5$, a flight distance (FD) lying in the expected range $(>500$ and $<2000 \mathrm{~mm})$ and to come from the IP. The muon identification efficiency versus 
the misidentification rate (misID) for different DLL cuts obtained on the calibration samples is shown in Fig. 3.

\section{Conclusion}

The latest station of the LHCb muon system is currently being installed while the other four stations are undergoing a commissioning phase. Tests performed on first beam data and using cosmic rays showed that the instrumented part of the detector is performing as expected and that is ready for the data taking foreseen in October. Monitoring and calibration procedures are in place and were tested in order to ensure that the best performances (L0 trigger and muon identification efficiencies) can be achieved since the very first running of the experiment.

\section{References}

[1] A.A. Alves, et al., J. Instr. 3 (2008) S08005, available at <http://www.iop.org/EJ/ journal/1748-0221

[2] V. Boccim, et al., IEEE Trans. Nucl. Sci. NS-55 (2008) 723.

[3] E. Polycarpo, M. Gandelman, The Performance of the LHCb Muon Identification Procedure, LHCb Note 2007-145, 2007. 